\title{
Reliability approach for fibre-reinforced composites design
}

\author{
H. Dehmous ${ }^{1,2}$, Hélène Welemane ${ }^{1, a}$, Moussa Karama ${ }^{1}$ and Kamel Aît Tahar ${ }^{2}$ \\ 1 Laboratory of Engineering Production, National Engineers School of Tarbes, 47 Avenue d'Azereix, BP 1629, \\ 65016 Tarbes, France \\ ${ }^{2}$ Laboratory of Mechanics and Structures, University of Tizi-Ouzou, Algeria
}

Received 8 March 2007, accepted 28 January 2008

\begin{abstract}
This study aims at investigating the mechanical reliability of unidirectional fibre-reinforced composites in view of their design optimization. Owing to the manufacturing process, many uncertainties affect inherently the properties of these materials. Besides, the brittle character of the fibre failure leads to important safety factors that limit their development for engineering applications. The objective is to get a realistic evaluation of the mechanical reliability of such structures which accounts for the various uncertainties involved. The effects of random design parameters on the composite failure are then investigated for different kinds of reinforcement (nature of fibre, mono-material or hybrid structures) with finite-element probabilistic solver PERMAS.
\end{abstract}

Key words: Composite materials; reliability engineering; mechanical failure; unidirectional fibre reinforcement; failure probability; sensitivity analysis

\section{Introduction}

Fibre-reinforced composites are gradually becoming more popular for several engineering applications and especially for mechanical structures by providing enhanced performances in comparison to classical materials and consequently new design perspectives [1]. Indeed, composite materials allow significant progress in weight-strength ratio, durability under thermal and fatigue solicitations and corrosion resistance which are of crucial interest in the fields of aeronautics (plane aileron, helicopter blade), transport (line shaft, suspension) or civil engineering (concrete pre-stressed cables, bridge suspension stays).

The counterpart of composite materials is related to the important scatter of their mechanical properties due to the manufacturing process. Besides, fibre materials (mostly used are carbon, Kevlar and glass) may exhibit a brittle behaviour for which failure occurs suddenly without critical signs. According to these uncertainties and risks, the traditional design of such structures takes into account important safety factors on fixed parameters (deterministic approach): at least a reduction of $50 \%$ of the characteristic strength for composite for only $30 \%$ in the case of steel. This severe safety margin generates a major increase of the structures dimensions and leads consequently to limiting costs for these design solutions.

In order to optimize this approach and then develop the use of composite materials for mechanical structures, it is essential to get a realistic evaluation of their reliability which especially accounts for the various uncertainties

\footnotetext{
a Corresponding author: Helene.Welemane@enit.fr
}

that affect their structural behaviour (material properties, loads and physical mechanisms of damage) [2-8]. In this way, reliability analysis offers a very useful tool in the conception phase as well as for the maintenance program:

- for structural design as it provides the range of use to achieve a specified reliability level, which consequently helps in the design optimization;

- for risk control on existing structures by evaluating the failure probability (equally the security level) and, if necessary, by defining the crucial parameters which mainly influence this phenomenon.

Such probabilistic analyses build a stochastic modelling that requires three important steps:

- first, the choice of input random variables that describe the various sources of uncertainties involved;

- then, the choice of a performance function (or failure criterion) which mathematically defines the structure failure;

- finally, the calculation of indicators (failure probability, failure index) which provide a quantitative and qualitative evaluation of the structure reliability.

In particular, the failure probability $P_{f}$ of a structure according to a vector $X$ of random variables representing the uncertainties in the model is defined by the following integral:

$$
P_{f}=\int_{G(X) \leq 0} f_{X}(X) d X
$$

where $f_{X}$ denotes the associated distribution function and $G$ is the performance function: $G(X)=0$ is the limit 


\begin{tabular}{|l|l|}
\hline$P_{f}$ & failure probability \\
\hline$\beta$ & reliability index \\
\hline$X$ & random variables vector \\
\hline$f_{X}$ & associated distribution function \\
\hline$G$ & performance function \\
\hline$N$ & number of evaluations of function $G$ \\
\hline$L$ & length of composite structure \\
\hline$\phi\left(\phi_{A}\right)$ & diameter (diameter of composite A in hybrid composite) \\
\hline$F$ & axial tension force in the longitudinal direction \\
\hline$q$ & uniform pressure in the transverse direction \\
\hline$J_{2}(\bar{\sigma})$ & second invariant of the stress tensor $\bar{\sigma}$ \\
\hline$F_{e}$ & initial plastic yield (elastic limit) \\
\hline$E_{i}$ & Young modulus in direction i \\
\hline$\nu_{i j}$ & Poisson ratio in plane ij \\
\hline$G_{i j}$ & Shear modulus in plane ij \\
\hline$\sigma_{1}, \sigma_{2}, \tau_{12}$ & stresses components in the principal material directions \\
\hline $\mathrm{T} 1$ & tension ultimate stresses in the fibre direction \\
\hline$T 2$ & tension ultimate stresses in the transverse direction \\
\hline$C 1$ & compression ultimate stresses in the fibre direction \\
\hline$C 2$ & compression ultimate stresses in the transverse direction \\
\hline$S$ & shear ultimate stresses \\
\hline
\end{tabular}

List of the symbols.

safety domain

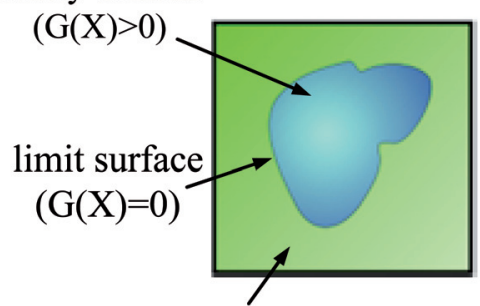

failure domain $(\mathrm{G}(\mathrm{X}) \leq 0)$

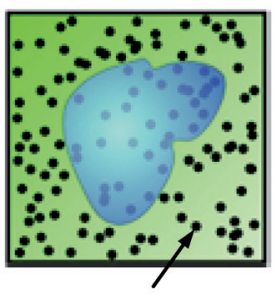

$\mathrm{N}$ evaluations of $\mathrm{G}(\mathrm{X})$

Fig. 1. Monte Carlo simulation method.

surface between the failure domain $(G(X) \leq 0)$ and the safety domain $(G(X)>0)$.

The mechanical systems considered usually exhibit a degree of complexity (many random variables whose joint density of probability $f_{X}$ is not available, integration domain with complex shape) that prevents the direct calculation of integral (1). Accordingly, various methods have been developed [9]:

- simulation methods (Monte Carlo for example) in which $N$ evaluations of function $G$ for various vectors realizations $X$ are performed and treated with statistical methods $\left(P_{f}\right.$ is defined as the ratio of failing cases to $N$, Fig. 1). Such method gives in all cases a good estimation of $P_{f}$ if the number of simulations $N$ is significant, which remains usually quite time expensive;

- approximation methods which represent the limit surface with linear (FORM) or second-order (SORM) polynomials to define the reliability index $\beta$, that is the shorter distance between the origin and the failure domain in the normalized space obtained through

an isoprobabilistic transformation (Fig. 2). Such approaches provide also the dependence of $\beta$ according to the different random variables, which is very interesting for the designer and for risk control.

This paper aims at associating a mechanical modelling with a reliability approach on the case of unidirectional fibre-reinforced composites. The objective is to investigate the nature of the composite material used and for each case, the impact of the variable design parameters on the structure failure.

\section{Structure description}

For this study, we consider a cylindrical composite structure (length $L=152 \mathrm{~mm}$, diameter $\phi=L / 10$ ) composed of an epoxy matrix reinforced by unidirectional fibres in the longitudinal direction (Fig. 3). The cylinder is embedded on one side and subjected to two loads (Fig. 4):

- an axial tension force $F$ in the longitudinal direction which represents the main solicitation of the structure;

- a uniform pressure $q$ in the transverse direction that we consider as a secondary load.

In a civil engineering context, such a structure is for example representative of a bridge stay submitted to axial tension through the bridge deck and to bending induced by lateral wind.

In order to investigate the reinforcement nature, the reliability analysis is performed for three different constitutive materials:

- steel as a reference;

- composite materials with a single kind of fibre reinforcement; 

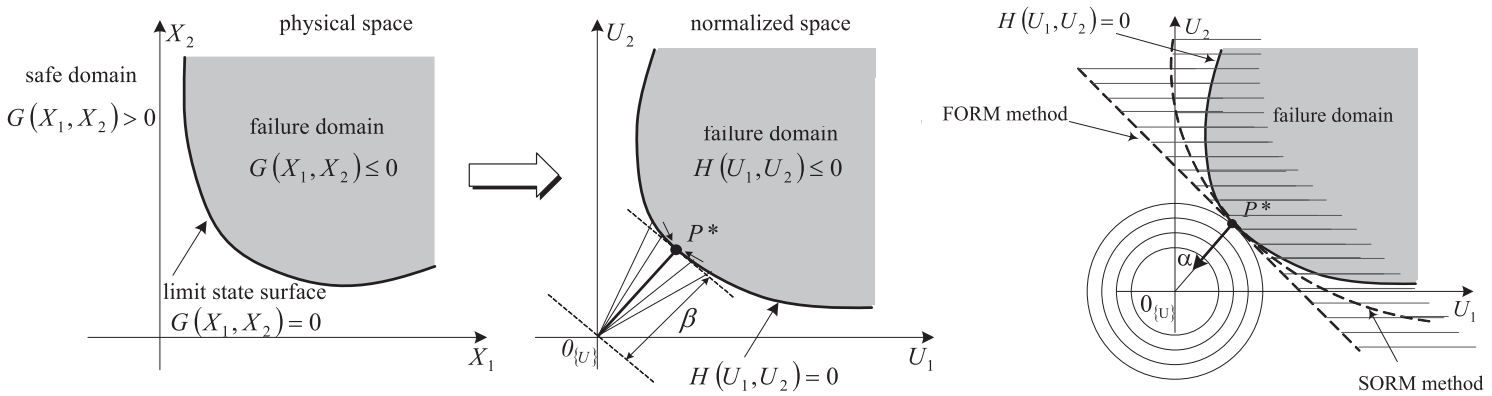

Fig. 2. Definition of the reliability index $\beta$ and approximation methods FORM/SORM [9].

Table 1. Elastic properties of fibre-reinforced composites (epoxy matrix).

\begin{tabular}{lccc}
\hline Nature of fibre & carbon & glass & Kevlar \\
& HRT800 & R & 49 \\
\hline Elastic modulus $E_{1}$ in fibre direction $(\mathrm{GPa})$ & 135 & 52 & 72 \\
Elastic modulus $E_{2}=E_{3}$ in transverse & 10 & 13.8 & 5.5 \\
direction $(\mathrm{GPa})$ & & & \\
Poisson ratio $\nu_{12}=\nu_{13}$ & 0.34 & 0.25 & 0.34 \\
Poisson ratio $\nu_{23}$ & 0.4 & 0.4 & 0.4 \\
Shear modulus $G_{12}=G_{13}(\mathrm{GPa})$ & 5 & 4.5 & 2.1 \\
\hline
\end{tabular}

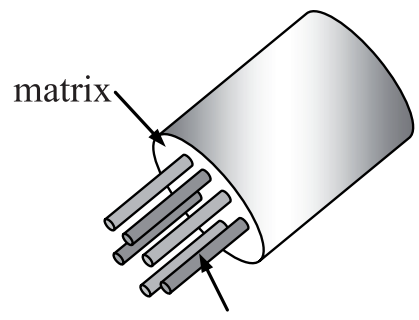

\section{Unidirectional fibres}

Fig. 3. Composite structure.

- hybrid material composed of two single fibrereinforced composites.

We consider here an homogeneous isotropic elasto-plastic steel with density of 7.85. According to the low variability observed during experimental results, mechanical properties take the following fixed values:

- Young modulus: $E=200 \mathrm{GPa}$;

- Poisson ratio: $\nu=0.3$;

- initial plastic yield (elastic limit) observed in an uniaxial tension test: $F_{e}=1600 \mathrm{MPa}$.

All composite materials are composed of $60 \%$ by volume of different unidirectional fibres in an epoxy resin matrix:

- carbon (density 1.6);

- glass (density 2);

- Kevlar (density 1.38).

Within the framework of this study, homogenized properties of these composite materials are considered (macroscopic description). As a matter of fact, they exhibit isotropic transverse anisotropy with axis of symmetry the fibre direction 1 , then direction 2 = direction 3 (Fig. 5).
Assuming stable values for elastic properties, data related to the three composite materials are specified in Table 1 [10].

The case of an hybrid structure made up of two coaxial cylinders (denoted A and B) composed of fibre-reinforced composites with different kinds of fibre for each is also investigated. Both composite materials have the same fibre direction, namely the longitudinal direction of the cylinders (Fig. 6). The composite A with diameter $\phi_{\mathrm{A}}$ is embedded in the composite $\mathrm{B}$ with external diameter $\phi$, and adhesion between A and B is supposed to be perfect.

\section{Reliability model}

As already mentioned, the reliability analysis requires first the choice of the random variables. Here the geometry, boundary conditions (connections), steel properties and composites elastic properties are considered as fixed parameters. Within random variables, we include the loads $(F$ and $q$ ) and composites failure parameters that enter the failure criteria expressions (see Eq. (3)), as we consider that they play a crucial role in the structure reliability. The probabilistic model retained for each random variable should derive in practice from statistical studies carried out on sufficiently representative data. In this paper, the probabilistic data are inspired by the bibliography devoted to the composites [10]. Accordingly, all the random parameters used for simulations are considered as lognormal distributed random variables, characterized by a mean value and a standard deviation defined in Table 2 .

The second stage consists in choosing a mathematical representation of the materials failure. In the case of steel, the failure domain is defined as the initial plastic domain 

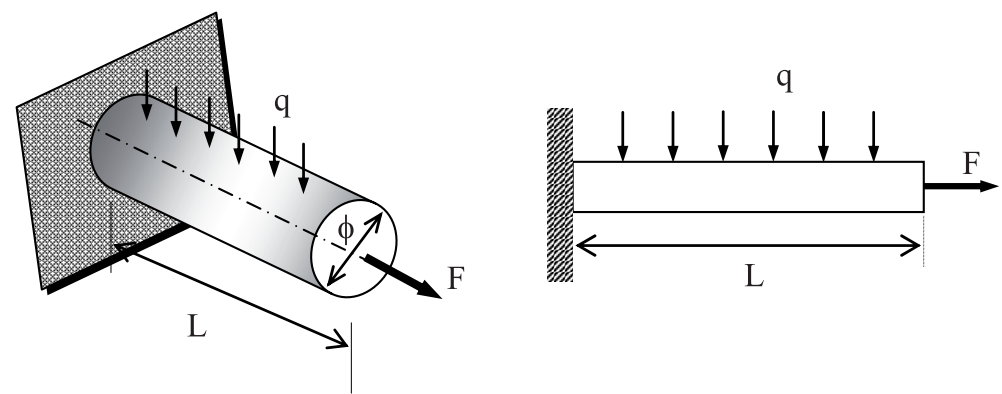

Fig. 4. Structure and loads.

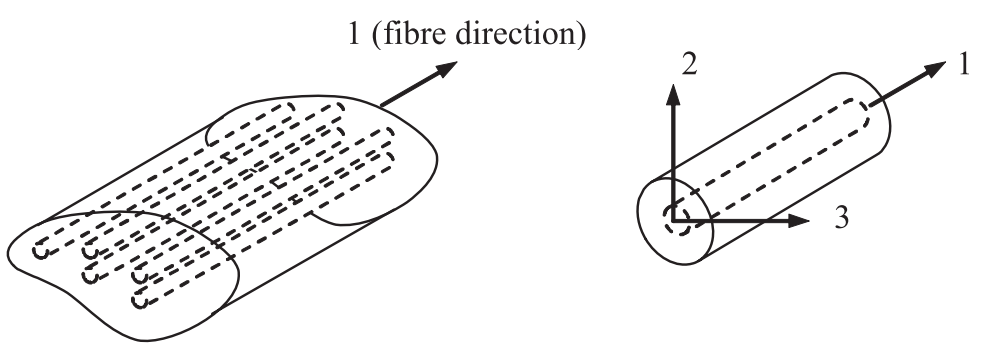

Fig. 5. Principal material coordinate system for unidirectional composite.

Table 2. Random variables.

\begin{tabular}{ccccc}
\hline & $\begin{array}{c}\text { Random } \\
\text { variables }\end{array}$ & \multicolumn{3}{c}{ Mean value } \\
\hline \multirow{2}{*}{ Loads } & $F$ & \multicolumn{2}{c}{ Various mean values investigated } \\
\hline Composites & $q$ & carbon-epoxy & glass-epoxy & Kevlar-epoxy \\
& $T 1(\mathrm{MPa})$ & 2550 & 1900 & 1380 \\
& $C 1(\mathrm{MPa})$ & 1470 & 970 & 280 \\
& $T 2(\mathrm{MPa})$ & 60 & 41 & 41 \\
& $C 2(\mathrm{MPa})$ & 270 & 138 & 138 \\
$S(\mathrm{MPa})$ & 100 & 70 & 60 \\
\hline
\end{tabular}

The standard deviation is equal to $10 \%$ for all random variables.

and described through the Von Mises criterion:

$$
G(\overline{\bar{\sigma}})=1-\frac{J_{2}(\overline{\bar{\sigma}})}{F_{e}}
$$

where $J_{2}(\overline{\bar{\sigma}})$ denotes the second invariant of the stress tensor $\overline{\bar{\sigma}}$. For composite materials, many criteria for unidirectional brittle elastic composite materials have been proposed in the literature $[11,12]$. In this study, reliability analysis is performed with the following Tsai-Wu criterion $[7,8]$ which allows to account for different behaviour in tension and compression:

$$
\begin{array}{r}
\mathrm{G}(\overline{\bar{\sigma}})=1-\frac{\sigma_{1}^{2}}{T 1 \cdot C 1}+\frac{\sigma_{2}^{2}}{T 2 \cdot C 2}+\frac{\tau_{12}^{2}}{S^{2}}-\frac{\sigma_{1} \sigma_{2}}{\sqrt{T 1 \cdot C 1 \cdot T 2 \cdot C 2}} \\
+\left(\frac{1}{T 1}-\frac{1}{C 1}\right) \sigma_{1}+\left(\frac{1}{T 2}-\frac{1}{C 2}\right) \sigma_{2}
\end{array}
$$

where $\sigma_{1}, \sigma_{2}$ and $\tau_{12}$ are the stresses components in the principal material directions, $T$ and $C$ denote respectively the tension and compression ultimate stresses (index 1 corresponds to the fibre direction, index 2 to the

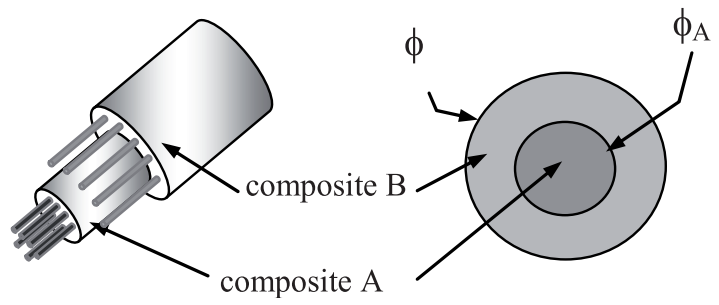

Fig. 6. Hybrid structure.

transverse direction), and $S$ represents the shear ultimate stress related to directions $1-(2=3)$.

\section{Simulation procedure}

Like mostly mechanical studies, the formulation of the limit state function $G$ is here implicit as the stress state $\overline{\bar{\sigma}}$ depends on the vector $X$ of random variables. Only particular evaluations of this function can be obtained by 


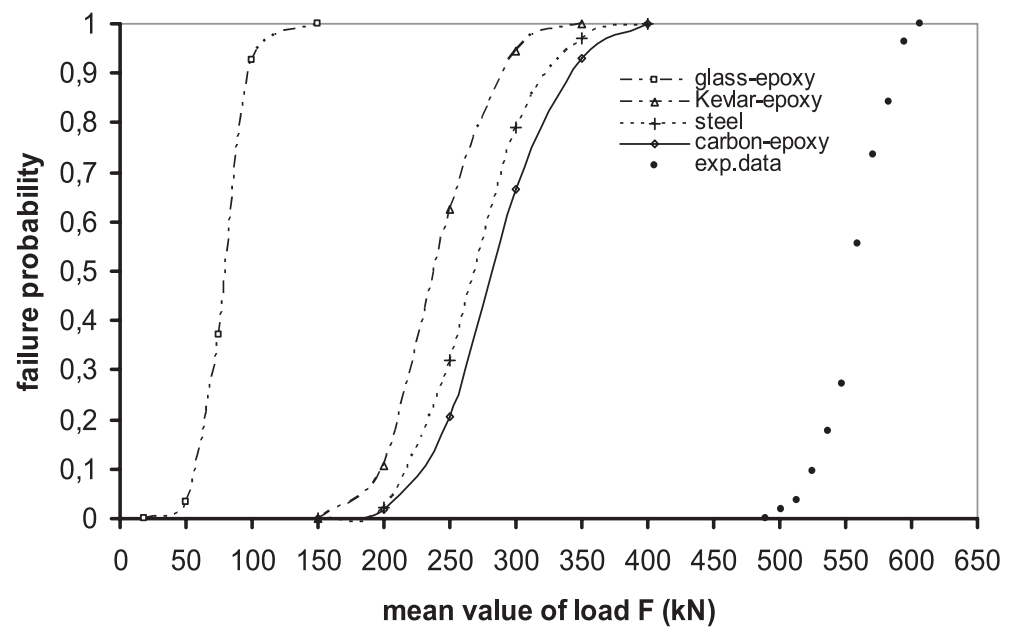

Fig. 7. Failure probability according to axial load $F(q=0 \mathrm{MPa})$.

numerical means, generally through finite element simulations. In order to perform the reliability analysis, it is then necessary to couple two numerical solvers each dedicated to a particular role:

- a probabilistic solver that generates the random variables vector realizations, carries out the isoprobabilistic transformation and finally validates the calculation steps and convergence to obtain the failure probability, reliability index $\beta$ and sensitivity analysis;

- a finite element solver that provides on the other hand the evaluations of the limit state function $G$ and its gradients.

In the present case, we use the PERMAS coupled code (see [13] for a review) which associates in a single numerical tool these two aspects. Such solver uses the Rosenblatt isoprobabilistic transformation and the classical HasoferLind-Rackwitz-Fiessler convergence algorithm to determinate the reliability index. In view of the quadratic form of the failure criteria retained, the SORM approximation method is used here to perform the reliability calculations. Note finally that the expression of the limit state function $G$ is introduced through a subroutine written in FORTRAN.

\section{Results and discussion}

The objective is to quantify here the effects of the random variables retained (loads, materials failure parameters) on the mechanical response of the structure and then to provide design recommendations to enhance its reliability.

\subsection{Mono-material structure}

Let examine first the case of the single material structure, either made of steel or single fibre-reinforced composite. Figure 7 shows the failure probability for the four materials studied (steel, carbon-epoxy, glass-epoxy and
Kevlar-epoxy) when the structure is subjected only to the axial force $F$ (pressure $q=0 \mathrm{MPa}$ ). For example for a load $F=200 \mathrm{kN}$, the probability that the carbon-epoxy, steel and Kevlar-epoxy structures fail are respectively $2 \%$, $2.25 \%$ and $10.5 \%$. On the other hand, the glass-epoxy composite has $100 \%$ chances of failing. If experimental data related to the composites investigated in this paper are not available, tests results obtained for another carbon epoxy composite (fibre volume ratio of $67 \%$, mean elastic modulus $E_{1}=155 \mathrm{GPa}$, mean tension ultimate stress in fibre direction $T 1=3075 \mathrm{MPa}$ [14]) are however represented in Figure 7 for comparison. We can note that the model seems to predict correctly the failure probability trend as the carbon composite studied here exhibits lower reinforcement ratio and mechanical performances (see Sect. 2).

For a given reliability level, one can compares the maximum force admissible for each material: taking steel as a reference, carbon-epoxy can support 5\% more, Kevlarepoxy $12 \%$ less and glass-epoxy $70 \%$ less for a failure probability $P_{f}=0.5$. The failure mode of the carbonepoxy and Kevlar-epoxy composites under longitudinal tension is induced by the fibre failure. Considering the properties of carbon and Kevlar fibers, the carbon-epoxy composite presents logically better performances. For glass-epoxy composite, failure mode is controlled by the transverse failure of matrix, accordingly this composite presents weak properties compared to others.

Figure 8 represents the evolution of the reliability in$\operatorname{dex} \beta$ according to $F$. Specific to approximation methods, such index constitutes another reliability indicator that can be linked to the failure probability [9]: precisely, more $\beta$ is high for a specified load, more the structure is reliable. For example for a load $F=200 \mathrm{kN}$, reliability index of carbon-epoxy, steel and Kevlar-epoxy structures are respectively $3.19,2.53$ and 1.25 . Carbon-epoxy composite presents then the highest reliability level, which confirms the tendency observed in Figure 7.

It is also interesting to investigate the effect of lateral pressure $q$ on the structure reliability. Figure 9 illustrates 


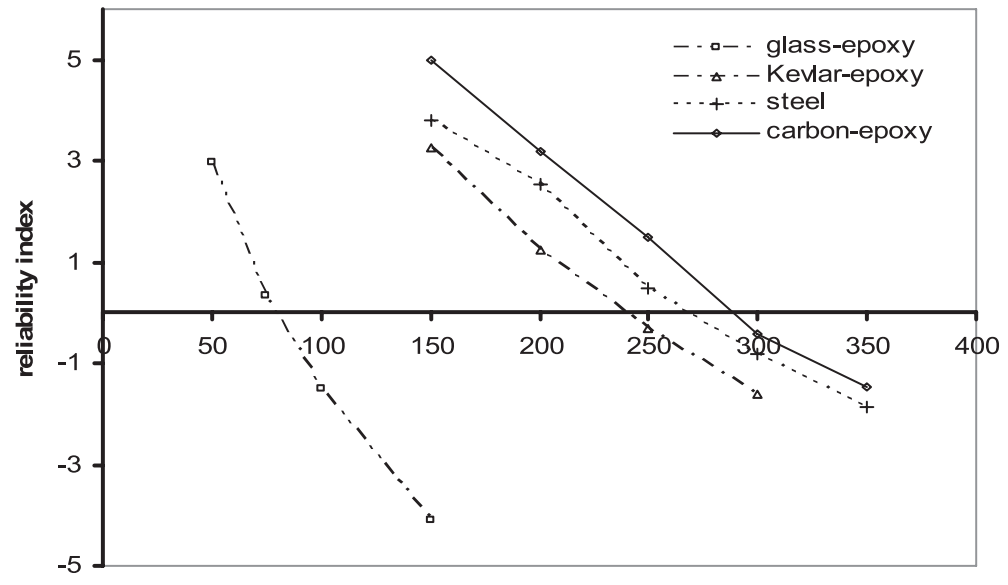

mean value of load $F(k N)$

Fig. 8. Reliability index $\beta$ according to axial load $F(q=0 \mathrm{MPa})$.

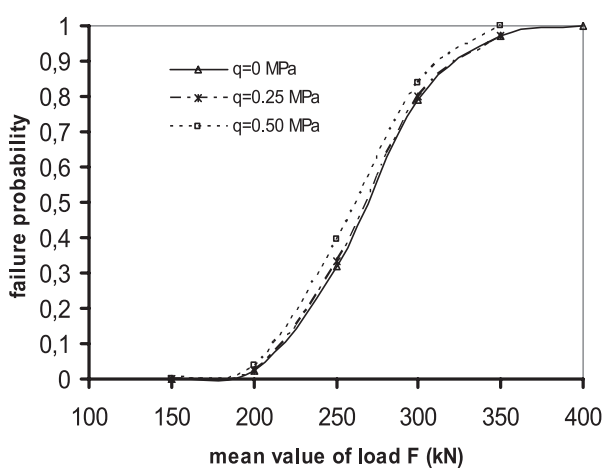

(a)

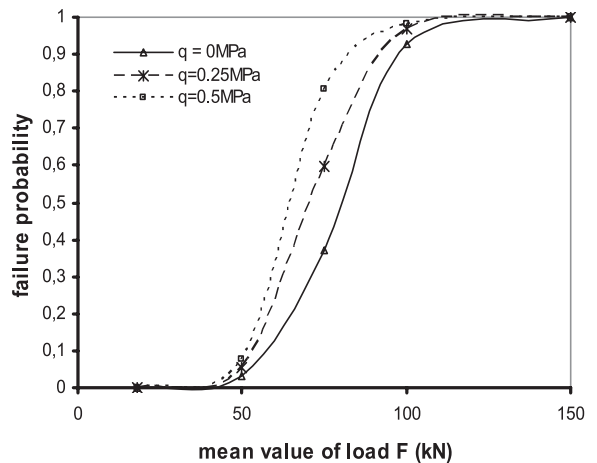

(c)

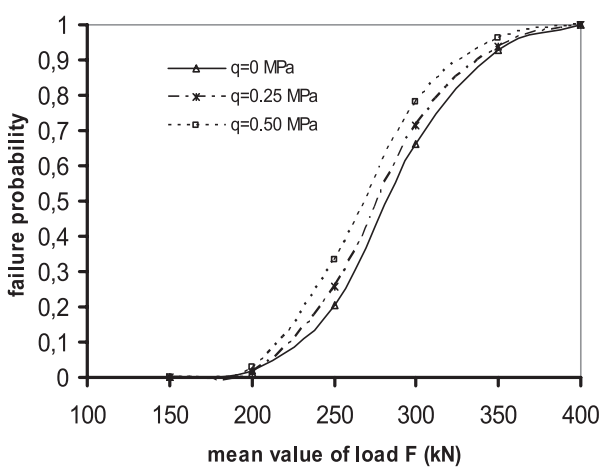

(b)

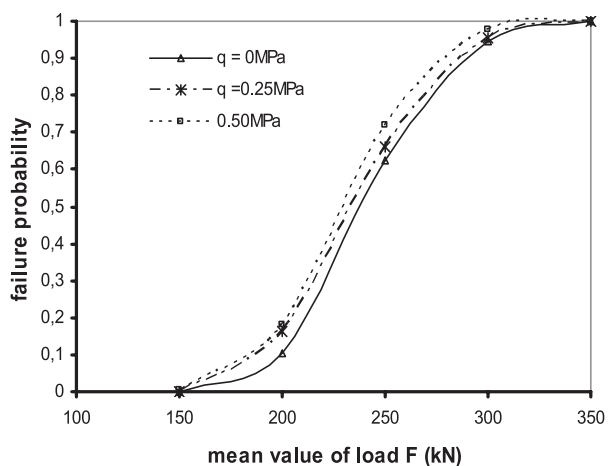

(d)

Fig. 9. Failure probability according to loads $F$ and $q$ : (a) steel, (b) carbon-epoxy, (c) glass-epoxy, (d) Kevlar-epoxy.

such impact for the different materials with failure probabilities according to loads $F$ and $q$. Note that values of $q$ are taken such that the axial stress induced by $q$ in the cylinder remains inferior to the one generated by load $F(\leq 400 \mathrm{kN})$. We observe in all cases that an increase of $q$ makes the structure less reliable. For example, the load $F$ associated to a probability of failure $P_{f}=0.5$ when $q=0 \mathrm{MPa}$ leads respectively to $P_{f}=0.58$ for steel, $P_{f}=0.65$ for carbon-epoxy, $P_{f}=0.88$ for glassepoxy and $P_{f}=0.6$ for Kevlar-epoxy when $q=0.5 \mathrm{MPa}$. Kevlar-epoxy appears then as the most stable composite according to the lateral load and should be used when many uncertainties may occur on this point.

In order to highlight the influence of composite failure parameters, a sensitivity study of index $\beta$ according to the mean value (Fig. 10) and to the standard deviation (Fig. 11) of these random variables is carried out for $P_{f} \approx 10^{-2}$ : the impact of the average values provides an indication for the more safety range of use, and the effect of the standard deviation underlines the role of the quality control. In particular, when the sensitivity factor of a parameter is positive (respectively negative), it 


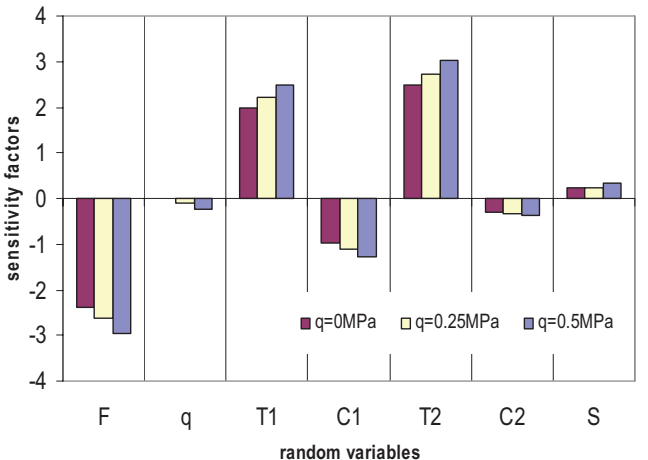

(a)

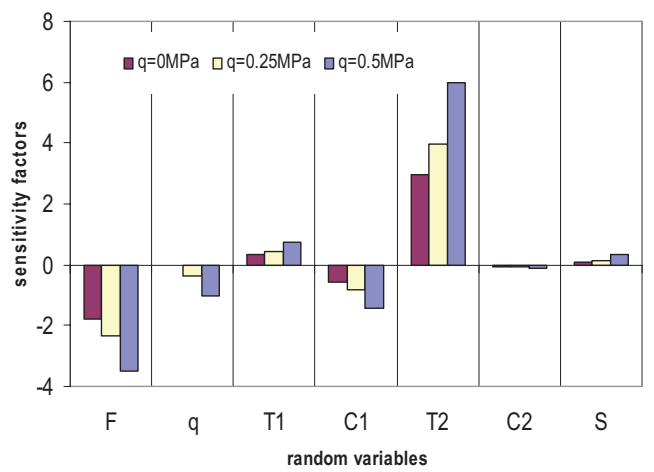

(b)

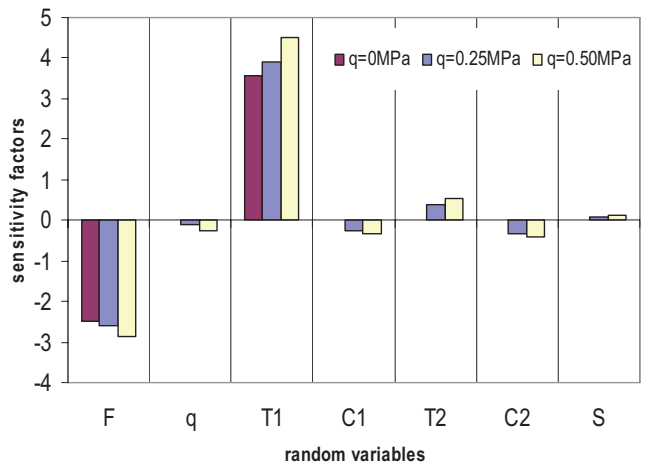

(c)

Fig. 10. Sensitivity analysis with respect to mean value of random variables $\left(P_{f} \approx 10^{-2}\right)$ : (a) carbon-epoxy, (b) glassepoxy, (c) Kevlar-epoxy.

means that an increase of this parameter increases (resp. decreases) reliability. Note finally that PERMAS solver performs each sensitivity analysis on the most probable failure point of the structure, which is generally close to the structure embedded side.

In Figure 10, we observe again that in all cases an increase of both loads tends to decrease the reliability, especially axial load $F$. The failure parameters influence is quite different for the three composites and an increase of the lateral load $q$ just accentuates their trends. In particular, the variables favourable to reliability are the tension ultimate stresses in fibre direction $(T 1)$ and transverse direction (T2): $T 1$ and $T 2$ for carbon-epoxy, $T 2$ for glassepoxy and $T 1$ for Kevlar-epoxy. To understand these re-

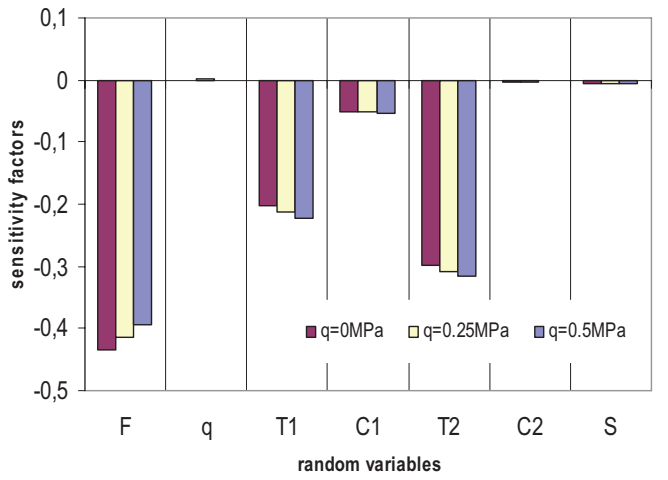

(a)

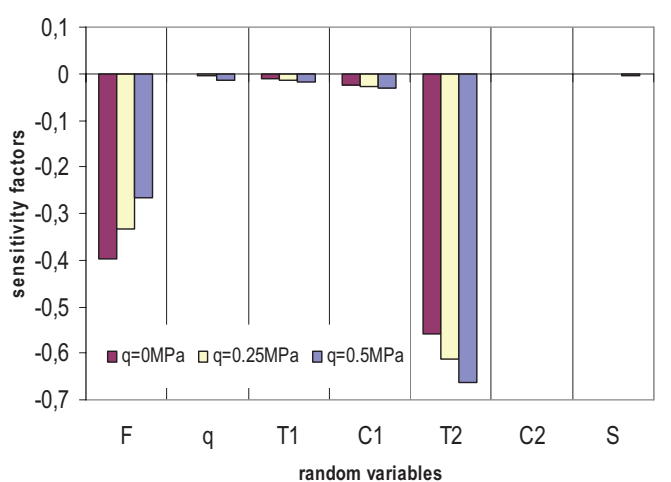

(b)

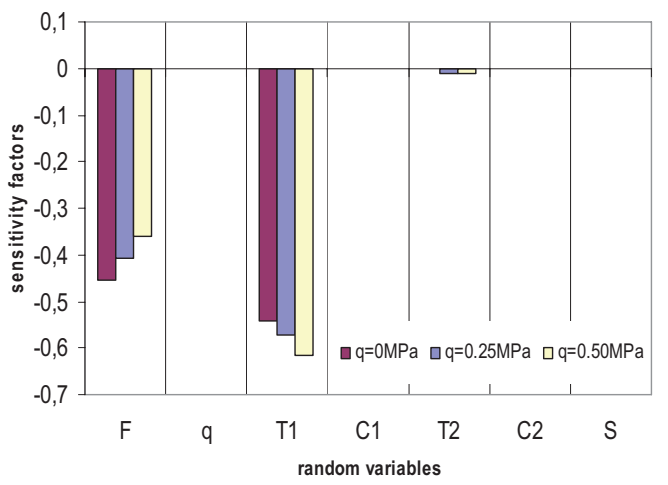

(c)

Fig. 11. Sensitivity analysis with respect to standard deviation of random variables $\left(P_{f} \approx 10^{-2}\right)$ : (a) carbon-epoxy, (b) glass-epoxy, (c) Kevlar-epoxy.

sults, stress components $\left(\sigma_{11}, \sigma_{22}\right.$ and $\tau_{12}$ in the principal material coordinate system) induced in the carbonepoxy structure are evaluated through deterministic calculations corresponding to $P_{f} \approx 10^{-2}$ (Tab. 3). For all values of pressure $q$, the stress state belongs to the tension domain $\left(\sigma_{1} \geq 0, \sigma_{2} \geq 0\right)$. Furthermore, the previously mentioned favourable parameters correspond for each material to the respective ultimate stresses (mean value defined at Tab. 2) that stresses $\sigma_{1}, \sigma_{2}$ or $\tau_{12}$ are closer to. Besides, the Tsai-Wu failure criterion applied to carbon-epoxy composite is drawn in the stress space $\left(\sigma_{1}, \sigma_{2}\right)$ for different values of limit stresses $T 1$ (mean value given at Tab. $2 \pm 10 \%$, Fig. 12). In the tension domain, we see that an increase of $T 1$ tends actually to 


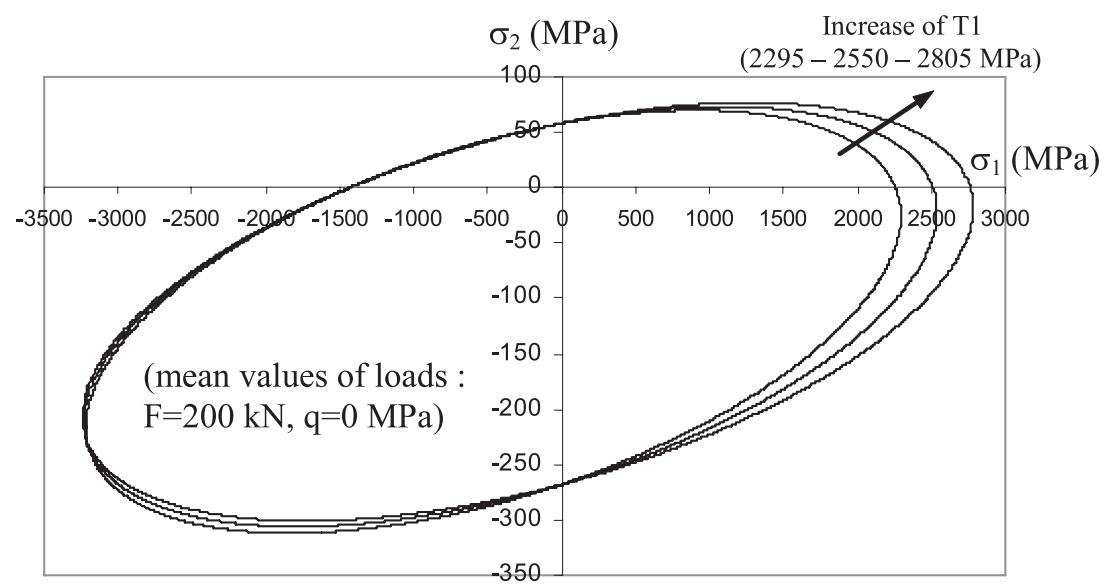

Fig. 12. Failure criteria according to the limit stress $T 1$ (carbon-epoxy).

Table 3. Deterministic calculations : stress ratios for carbonepoxy $(F=200 \mathrm{kN})$.

\begin{tabular}{lccc}
\hline & $\sigma_{1} / T 1(\%)$ & $\sigma_{2} / T 2(\%)$ & $\tau_{12} / S(\%)$ \\
\hline$q=0 \mathrm{MPa}$ & 43.6 & 77.7 & 20.1 \\
$q=0.25 \mathrm{MPa}$ & 45.2 & 80.7 & 20.3 \\
$q=0.5 \mathrm{MPa}$ & 46.8 & 83.5 & 22.2 \\
\hline
\end{tabular}

extent the safety domain, and then to reduce the failure probability.

Sensitivity factors related to the standard deviation of all random variables are negative which confirms the idea that the variability of a parameter reduces the structure reliability. The minimization of uncertainties by a good systematic quality control is then essential for the structure assessment. Figure 11 shows the respective effect for each random parameter and follows the trends observed for the sensitivity analysis on mean values. Reliability is then essentially improved when the axial load $F$ and tension ultimate stresses are estimated with the best precision ( $T 1$ and $T 2$ for carbon-epoxy, $T 2$ for glass-epoxy and $T 1$ for Kevlar-epoxy).

\subsection{Hybrid structure}

In this part, we focus our attention on composite materials that lead previously to the best performances, namely the carbon-epoxy and Kevlar-epoxy. The internal diameter is fixed to $\phi_{\mathrm{A}}=\phi / 2$ and two configurations are considered:

- hybrid I: carbon-epoxy inside (A), Kevlar-epoxy outside (B);

- hybrid II: Kevlar-epoxy inside (A), carbon-epoxy outside $(\mathrm{B})$.

To study the reliability of such hybrid structures, we adopt the same approach as [8] by evaluating the failure probability of each single fibre-reinforced composite (A and B) within the structure. This is an independent analysis of both parts of the structure which do not take into

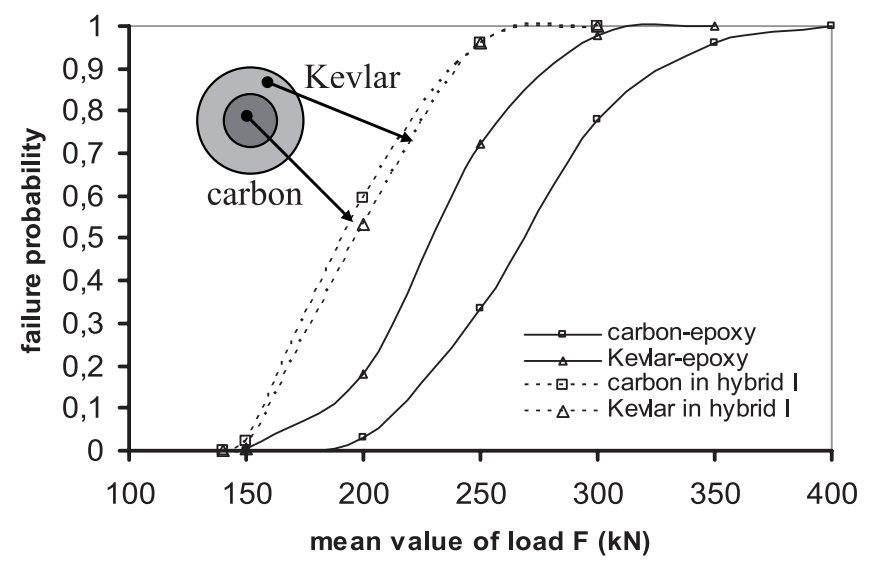

Fig. 13. Failure probability of hybrid I according to axial load $F(q=0.5 \mathrm{MPa})$.

account any load transfer when one part fails. Such study provides then the weakest composite material (between $\mathrm{A}$ and $\mathrm{B}$ ) within the hybrid structure which exhibits the biggest failure probability for a given load. Accordingly, if we consider that the failure of the structure occurs when one layer fails, the structure reliability is then directly given by the curve relative to the weakest material. The failure probabilities of hybrid I (Fig. 13) and hybrid II (Fig. 14) according to axial load $F$ are presented with the previous results on mono-material cylinders (with diameter $\phi$ ) for each composite as a reference.

We can point out that configuration $I$ is much more critical than composite material alone as $P_{f}$ is much more important in both Kevlar-epoxy and carbon-epoxy parts. Especially, this geometry leads to extra stresses causing failure of carbon-epoxy sooner. Looking at the other configuration, we note that hybrid II allows an improvement as the failure probability of carbon-epoxy in the structure decreases slightly. It may then be interesting to investigate such design option to optimise the structure strength. 


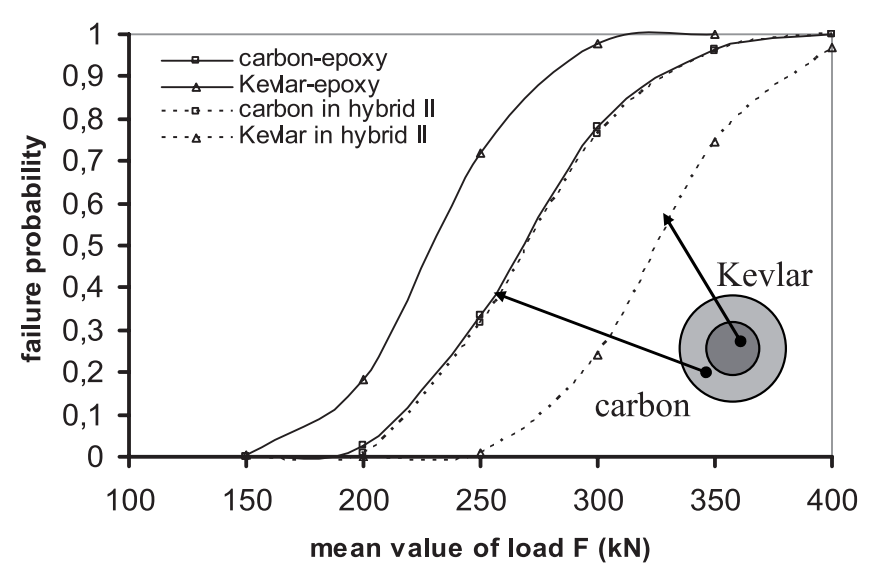

Fig. 14. Failure probability of hybrid II according to axial load $F(q=0.5 \mathrm{MPa})$.

\section{Conclusion and perspectives}

The objective of this study is to highlight the interest in introducing composite materials into the field of engineering mechanical design. In this way, probabilistic simulations with finite element solver PERMAS are performed to analyse the mechanical reliability of unidirectional fibre-reinforced composite structures. The failure probability of different kinds of single fibre reinforcement is then evaluated: carbon-epoxy exhibits the best performance related to axial load (better than steel), the behaviour of Kevlar-epoxy is particularly stable according to lateral pressure, whereas glass-epoxy is definitively weak on all these points. The sensitivity analysis provides also interesting information for the design optimisation, namely that ultimate tension stresses take a significant part and should be as big as possible to improve the structure reliability.

The results obtained on the hybrid composite seem attractive at first hand, especially as Kevlar fibres very much less expensive than carbon. However we suppose here a perfect adherence between the two layers of the composite. In the real case, it is thus possible that interface between these two layers fails. This point needs to be checked through experimental tests before further modelling investigations.

In order to complete this work, it may be interesting to look at the influence of the kind of statistical models introduced in the probabilistic solver and also to investigate others hybrid solutions such as mono-material composite made of epoxy resin reinforced by different kinds of fibres.

\section{References}

1. U. Meier, Carbon fibre reinforced polymers, Modern materials in bridge engineering. Structural Engineering International 1, 1-12 (1992).

2. C. Boyer, A. Béakou, M. Lemaire, Design of a composite structure to achieve a specified reliability level. Reliability Engineering and System Safety 56, 273-283 (1997).

3. S. Guedes, Reliability of components in composite materials. Reliability Engineering and System Safety 55, 171177 (1997).

4. M. Walker, R. Smith, A methodology to design fibre reinforced laminated composite structures for maximum strength. Composites Part B: Engineering 34, 200-214 (2003).

5. J.L. Pelletier, S.S. Vel, Multi-objective optimisation of fiber reinforced composite laminates for strength, stiffness and minimal mass. Computer and Structures 84, 20652080 (2006).

6. J. Renard, A. Thionnet, Damage in composites: From physical mechanisms to modelling. Composites Science and Technology 66, 642-646 (2006).

7. T.P. Philippidis, D.J. Lekou, Probabilistic failure prediction for FRP composites. Composites Science and Technology 58, 1973-1982 (1998).

8. D.M. Frangopol, S. Recek, Reliability of fiber reinforced composite laminate plates. Probabilistic Engineering Mechanics 18, 119-137 (2003).

9. O. Ditlevsen, H. Madsen, Structural reliability analyses. J. Wiley \& Sons (1996).

10. F.L. Matthews, R.D. Rawlings, Composite Materials: Engineering and Science. Woodhead Publishing Ltd and CRC Press LLC (2003).

11. A.S. Kaddour, M.J. Hinton, P.D. Soden, A comparison of the predictive capabilities of current failure theories for composite laminates: additional contributions. Composites Science and Technology 64, 449-476 (2004).

12. M.J. Hinton, A.S. Kaddour, P.D. Soden, Evaluation of failure prediction in composite laminates: background to 'part C' of the exercise. Composites Science and Technology 64, 321-327 (2004).

13. M.F. Pellissetti, G.I. Schuëller, On general purpose software in structural reliability. Structural Safety 28, 3-16 (2006).

14. H. Dehmous, H. Welemane, I. Zivanovic, M. Karama, Reliability analysis of composite structures: application to the Laroin footbridge, in Proceedings of ICSAM Conference. Patras, September (2007). 